\title{
Is kidney function associated with cognition and mood in late life?
}

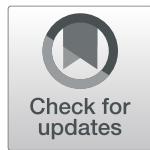

\section{The Screening for CKD among Older People across Europe (SCOPE) study}

\author{
Lisanne Tap ${ }^{1}$, Andrea Corsonello ${ }^{2}$, Francesc Formiga ${ }^{3}$, Rafael Moreno-Gonzalez ${ }^{3}$, Johan Ärnlöv ${ }^{4,5,6}$, \\ Axel C. Carlsson ${ }^{4,6}$, Regina Roller-Wirnsberger ${ }^{7}$, Gerhard Wirnsberger ${ }^{7}$, Gijsbertus Ziere ${ }^{1}$, Ellen Freiberger ${ }^{8}$, \\ Cornel Sieber ${ }^{8}$, Tomasz Kostka ${ }^{9}$, Agnieszka Guligowska ${ }^{9}$, Pedro Gil ${ }^{10}$, Sara Lainez Martinez ${ }^{10}$, \\ Rada Artzi-Medvedik ${ }^{11,12}$, Ilan Yehoshua ${ }^{12}$, Paolo Fabbietti ${ }^{2,13}$, Fabrizia Lattanzio ${ }^{2}$, Francesco Mattace-Raso ${ }^{1}$ and on \\ behalf of SCOPE investigators
}

\begin{abstract}
Background: Chronic kidney disease (CKD), cognitive impairment and depression share common risk factors. Previous studies did not investigate the possible association between kidney function and cognitive and mood disorders in older persons in a broad range of kidney function. The present study explored associations between kidney function, cognition and mood in outpatients of 75 years and over.
\end{abstract}

Methods: Baseline data of 2252 participants of the SCOPE study, an international multicenter cohort observational study, were used in which community-dwelling persons of 75 years and over were enrolled to screen for CKD Kidney function was estimated with the BIS1-eGFR equation, cognition was assessed with the Mini-Mental State Examination (MMSE) and mood with the Geriatric Depression Scale 15 items (GDS-15). Characteristics were compared across stages of CKD. Mean eGFR values were also compared across categories of MMSE $(<24,24-26$, 227) and between groups with high and low score on the GDS-15 (>5/ $\leq 5)$.

Results: In total, $63 \%$ of the population had an eGFR $<60 \mathrm{~mL} / \mathrm{min}$. In advanced stages of CKD, participants were older and more often men than in earlier stages $(p<0.001)$. Cardiovascular diseases and diabetes mellitus were more often found in those in advanced stages of CKD $(p<0.001)$, and also cumulative comorbidity scores were higher than in those in earlier stages $(p<0.001)$. Median MMSE was 29 in CKD stage $1-2$ and 3 , and 30 in CKD stage 4, whereas median GDS-15 score was 2 in all stages of CKD. Mean values of eGFR did not differ across categories of MMSE or between groups with high and low score on the GDS-15. Stratification for albuminuria did not change these results.

(Continued on next page)

\footnotetext{
* Correspondence: a.corsonello@inrca.it

${ }^{2}$ Italian National Research Center on Aging (IRCCS INRCA), Ancona, Fermo and Cosenza, Italy

Full list of author information is available at the end of the article
}

\section{$\triangle B M C$}

(c) The Author(s). 2020 Open Access This article is licensed under a Creative Commons Attribution 4.0 International License, which permits use, sharing, adaptation, distribution and reproduction in any medium or format, as long as you give appropriate credit to the original author(s) and the source, provide a link to the Creative Commons licence, and indicate if changes were made. The images or other third party material in this article are included in the article's Creative Commons licence, unless indicated otherwise in a credit line to the material. If material is not included in the article's Creative Commons licence and your intended use is not permitted by statutory regulation or exceeds the permitted use, you will need to obtain permission directly from the copyright holder. To view a copy of this licence, visit http://creativecommons.org/licenses/by/4.0/ The Creative Commons Public Domain Dedication waiver (http://creativecommons.org/publicdomain/zero/1.0/) applies to the data made available in this article, unless otherwise stated in a credit line to the data. 
(Continued from previous page)

Conclusions: Older persons in more advanced stages of CKD did not have lower cognitive scores or higher rates of depressive symptoms than older persons in earlier stages. Future longitudinal studies might give information on the possible effect of kidney function on cognition and mood in late life.

Trial registration: This study was registered prospectively on 25th February 2016 at clinicaltrials.gov (NCT02691546).

Keywords: Chronic kidney disease, Estimated glomerular filtration rate, Cognition, Cognitive impairment, Mood, Depressive symptoms, Older persons

\section{Background}

The prevalence of chronic kidney disease (CKD), cognitive impairment and cardiovascular conditions is growing as result of the aging population [1-3]. CKD, cognitive impairment and mood disorders can share common risk factors, such as hypertension and diabetes mellitus $[1,2,4,5]$. However, whether a decreased kidney function is associated with cognitive and mood disorders in the oldest old is not completely clear.

One of the potential mechanisms underlying the interaction between the kidney and the brain may include the presence of small vessel disease [6]. Persons with CKD have a higher burden of traditional vascular risk factors such as hypertension, diabetes mellitus and dyslipidaemia which are related to small vessel disease in the kidney $[6,7]$. The same risk factors are also associated with cerebral white matter lesions, microbleeds, lacunar infarcts and subcortical atrophy which are markers of cerebral small vessel disease increasing the risk of stroke, cognitive decline and dementia [6, 8-11]. Moreover, the presence of small vessel disease may also underlie the association between CKD and depressive symptoms as result of a disruption of brain structures and connecting pathways in mood regulation [12]. Both cognition and mood may also be influenced by metabolic dysregulation and direct effects of lower glomerular filtration rate (GFR) [13-15].

Previous studies suggest that CKD and cognitive impairment might be correlated [16]. However, most studies did not focus on older patients in a broad range of GFR. In addition, in people in all stages of CKD, it was found that the prevalence of depressive symptoms ranged from 7 to $50 \%$ and depressive symptoms were more frequent in people in advanced stages of CKD than in people in earlier stages [17]. Nonetheless, most study populations were relatively small and studies did not focus on older persons. Therefore, the relationship between kidney function, cognition and mood in late life remains undetermined.

Since the identification of modifiable risk factors of cognitive and functional decline such as CKD is relevant, this study aimed to investigate the possible association between kidney function, cognition and mood in outpatients aged 75 years and over. We hypothesized that cognitive impairment and depressive symptoms would be more prevalent in advanced stages of CKD than in earlier stages and that persons with cognitive impairment and depressive symptoms would have lower levels of estimated GFR (eGFR) than persons without. Results of this study might provide important information in addition to known risk factors of adverse outcomes in persons with poor kidney function, cognitive impairment and depression. These older persons might then benefit from improved therapeutic strategies when visiting their nephrologist, geriatrician or psychologist.

\section{Methods}

The present study was performed within the framework of the Screening for Chronic Kidney Disease among Older People across Europe (SCOPE) study. The SCOPE study (European Grant Agreement no. 436849), is a multicenter 2-year prospective cohort study involving patients older than 75 years attending outpatient services in participating institutions in Austria, Germany, Israel, Italy, The Netherlands, Poland and Spain. Methods of the SCOPE study have been extensively described elsewhere [18]. The primary objective of the SCOPE study was to investigate the currently available screening methods to identify community-dwelling older patients at risk of kidney disease. Patients with end-stage renal disease or dialysis, a history of solid organ or bone marrow transplantation, an active malignancy or metastatic cancer within 24 months prior to the visit, a life expectancy of less than 6 months, a severe cognitive impairment or patients unwilling to provide consent were ineligible for the SCOPE study. Participants were requested to sign a written informed consent before entering the study. The study protocol was approved by ethics committees at all participating institutions, and complies with the Declaration of Helsinki and Good Clinical Practice Guidelines. Only baseline data are used in the present study. Overall, 2461 participants were initially enrolled in the study.

\section{Kidney function}

Serum creatinine (Isotope-Dilution Mass Spectrometry traceable) and albumin-to-creatinine ratio (ACR) were measured at local level by standard methods. Creatinine- 
based eGFR was calculated in $\mathrm{mL} / \mathrm{min} / 1.73 \mathrm{~m}^{2}$ using the Berlin Initiative Study 1 (BIS1) equation [19]: $3736 \times$ creatinine $^{-0.87} \times$ age $^{-0.95} \times 0.82$ (if woman).

The prevalence of stages of CKD was obtained using the Kidney Disease Improving Global Outcomes (KDIGO) guidelines [20]: eGFR $\geq 60$, stage $1-2$; 59.9-45, stage 3a; $44.9-30$, stage $3 \mathrm{~b}$; and $15-30 \mathrm{~mL} / \mathrm{min} / 1.73 \mathrm{~m}^{2}$, stage 4 . Albuminuria was defined as a urine ACR $\geq 30 \mathrm{mg} / \mathrm{g}$ ( $\geq 3$ $\mathrm{mg} / \mathrm{mmol}$ ) [20].

\section{Cognition}

The cognitive functions were measured by the Mini-Mental State Examination (MMSE) [21]. The MMSE is the most commonly administered psychometric screening tool of cognitive functioning and available and validated in all languages of participating countries. The score ranges from 0 to 30 points, whereas a score $<24$ is frequently implemented as the cut-off value for abnormal and indicative of cognitive impairment [22]. A meta-analysis of the accuracy of the MMSE in the detection of dementia and mild cognitive impairment showed a pooled sensitivity and specificity of 71.1 and $95.6 \%$ respectively in mixed specialist hospital settings [23]. A MMSE score of $<27$ might identify those with a greater risk of cognitive dysfunction, especially in highly educated persons [24]. Therefore, in this study, we defined 3 categories based on the MMSE score: $\geq 27,24-26$ and $<24$.

\section{Mood}

The 15-item Geriatric Depression Scale-Short Form (GDS), available and validated in all languages of participating countries, was used to investigate depressive symptoms [25]. It focusses on functional and mood symptoms of depression. The score ranges from 0 to 15 points, with higher scores indicating more depressive symptomatology. The cut-off score most often used for this GDS version is 5 or 6 [26]. A recent systematic review and meta-analysis showed a pooled sensitivity and specificity of 86 and $79 \%$, respectively to detect depression in older persons [27]. In this study, a score $>5$ was seen as suggestive of depressive symptoms [25].

\section{Other variables}

Demographic data and socioeconomic status were documented. Information on alcohol use, smoking status, medical history and use of medication was collected and the cumulative illness rating scale for geriatrics (CIRS-G) was calculated in order to score the comorbidity burden by rating the severity of medical problems affecting various organ systems [28]. During the study visit, a comprehensive geriatric assessment (CGA) was performed including also information on basic activities of daily living (ADL) and instrumental ADL (IADL) [29, 30].

\section{Statistical analysis}

Descriptive statistics were expressed as percentage for categorical variables and median and interquartile ranges (IQR) for continuous non-normally distributed variables. First, characteristics were compared across stages of CKD using the Chi square test and Kruskal Wallis test. Second, the correlation between age, MMSE and GDS was explored using the Spearman's correlation test. Third, mean eGFR values were compared across categories of cognition (MMSE< 24, MMSE 24-26, MMSE $\geq 27$ ) using analysis of variance (ANOVA) in the total population and stratified for the presence of albuminuria. Then, mean eGFR values were also compared between participants with low and high score and GDS (score $\leq 5$ and $>5$ ) in the total population and stratified for the presence of albuminuria. A $p$-value of $<0.05$ was considered statistically significant.

\section{Results}

Overall, 2461 participants were initially enrolled in the SCOPE study. Of them, 209 participants had missing data on serum creatinine, MMSE and/or GDS leaving a final sample of 2252 participants to be included in the present study. Baseline characteristics are shown in Table 1. Median age was 79.5 years (IQR 77.1-82.9), $55.7 \%$ were women and $24.5 \%$ were living alone. The majority of the population had hypertension (76.8\%), $25.1 \%$ had diabetes mellitus, $17.2 \%$ had a history of malignancy and $16.6 \%$ had congestive heart failure. About 9\% had a history of transient ischemic attack and 5.8\% have had a stroke. The median total score on the CIRSG was 8 (IQR $5-11$ ). Only $0.6 \%$ of the participants had an eGFR $\geq 90 \mathrm{~mL} / \mathrm{min} / 1.73 \mathrm{~m}^{2}, 36.2 \%$ were classified as CKD stage 2, 38.3\% had CKD stage 3A, 18.6\% had CKD stage $3 \mathrm{~B}$ and $6.3 \%$ had CKD stage 4 . In $26.7 \%$ of the study population, albuminuria was present. Median score on the MMSE was 29 (IQR 27-30), 7.1\% of the participants had a MMSE score $<24$. Median score on the GDS was 2 (IQR 1-4), 14\% of the participants had a GDS score $>5$.

Characteristics stratified for stages of CKD (stage 1-2 merged) are presented in Table 2 . In advanced stages of CKD, participants were older, more often men and former smokers and had fewer years of education than in earlier stages. Comorbidities such as cardiovascular diseases (hypertension, atrial fibrillation, heart failure), diabetes mellitus and history of malignancy were more often found in those in advanced stages of CKD and the CIRS-G total score and severity index were higher than in those in earlier stages. In participants with CKD stage 1-2, 3a and 3b, median MMSE score was 29 (IQR 2730), whereas median MMSE score in participants with CKD stage 4 was slightly higher with a median value of 30 (IQR 28-30). The proportion of participants with an 
Table 1 Baseline characteristics $(n=2252)$

\begin{tabular}{|c|c|}
\hline Age, years & $79.5(77.1-82.9)$ \\
\hline Women, \% & 55.7 \\
\hline Living alone, \% & 24.5 \\
\hline Education, years & $11(8-15)$ \\
\hline \multicolumn{2}{|l|}{ Smoking status } \\
\hline Current smoker, \% & 4.4 \\
\hline Former smoker, \% & 39.5 \\
\hline Alcohol $\geq 1$ unit a day, $\%$ & 25.8 \\
\hline $\mathrm{BMI}, \mathrm{kg} / \mathrm{m}^{2}$ & $27.3(24.7-30.4)$ \\
\hline ADL-independent, $\%$ & 95.2 \\
\hline ¡ADL-independent, n \% & 56 \\
\hline MMSE, score & $29(27-30)$ \\
\hline MMSE < 24, \% & 7.1 \\
\hline GDS, score & $2(1-4)$ \\
\hline $\mathrm{GDS}>5, \%$ & 14 \\
\hline Hypertension, \% & 76.8 \\
\hline Diabetes mellitus, \% & 25.1 \\
\hline $\mathrm{TIA}, \%$ & 8.7 \\
\hline Stroke, \% & 5.8 \\
\hline Atrial fibrillation, \% & 15.3 \\
\hline COPD, \% & 11.8 \\
\hline Cancer, \% & 17.2 \\
\hline$C H F, \%$ & 16.6 \\
\hline Vascular disease, \% & 12.6 \\
\hline CIRS-G, total score & $8(5-11)$ \\
\hline CIRS-G, severity index & $1.5(1.2-1.8)$ \\
\hline eGFR-BIS, mL/min/1.73 m² & $55.6(45.1-64.4)$ \\
\hline 90 or more, $\%$ & 0.6 \\
\hline $60-89.9, \%$ & 36.2 \\
\hline $45-59.9, \%$ & 38.3 \\
\hline $30-44.9, \%$ & 18.6 \\
\hline 30 or less, $\%$ & 6.3 \\
\hline $\mathrm{ACR}, \mathrm{mg} / \mathrm{g}$ & $11.2(33.1-3.4)$ \\
\hline Albuminuria, \% & 26.7 \\
\hline
\end{tabular}

Values are expressed as percentage or median (IQR)

Abbreviations: BMI Body Mass Index, (i) $A D L$ (instrumental) Activities of Daily Living, MMSE Mini-Mental State Examination, GDS Geriatric Depression Scale, TIA Transient Ischemic Attack, COPD Chronic Obstructive Pulmonary Disease, CHF Congestive Heart Failure, CIRS-G Cumulative Illness Rating Scale for Geriatrics, eGFR-BIS Estimated Glomerular Filtration Rate, $A C R$ Albumin-to-Creatinine Ratio

MMSE score $<24$ did not differ across stages of CKD, with a prevalence of $6.4 \%$ (stage $1-2$ ), $7.7 \%$ (stage $3 \mathrm{~A}$ ), 9.3\% (stage 3B) and 5\% (stage 4). Median score on the GDS was the same in all stages of CKD, namely 2 (IQR 1-4). A GDS score $>5$ was found in 13, 15, 14.1 and $14.2 \%$ of the participants in each stage of CKD, respectively. A correlation was found between age and MMSE score (Spearman's rho $-0.229, p<0.001$ ) and age and GDS score (Spearman's rho 0.076, $p<0.001$ ).

Figure 1a shows mean values of eGFR across predefined categories of MMSE. One hundred and fifty nine participants had a MMSE score <24, 309 had a MMSE score of 24-26 and 1784 participants had a MMSE score $\geq 27$. Mean eGFR values did not differ across categories; mean values and 95\% CI were 53.5 (51.1-55.8), 55 (53.4-56.5) and $54.7(53.9-55.4) \mathrm{mL} / \mathrm{min} / 1.73 \mathrm{~m}^{2}$, respectively. Mean values of eGFR did not differ between participants with a low $(n=1936)$ and high score $(n=$ $316)$ on the GDS; mean values and $95 \%$ CI were 54.7 (54-55.3) and $54.3(52.6-55.9) \mathrm{mL} / \mathrm{min} / 1.73 \mathrm{~m}^{2}$, respectively. Results are shown in Fig. $1 \mathrm{~b}$.

In Fig. 2a mean values of eGFR in participants with and without albuminuria are shown across categories of MMSE. In participants with albuminuria, 45 participants had a MMSE score $<24,86$ had a MMSE score of 24-26 and 470 participants scored $\geq 27$. Mean values of eGFR did not differ across categories; mean values and 95\% CI were 47.1 (42-52.3), 47.1 (43.6-50.5) and 45.2 (43.746.8) $\mathrm{mL} / \mathrm{min} / 1.73 \mathrm{~m}^{2}$, respectively. In participants without albuminuria, the number of participants in the same categories were 114, 223 and 1314, respectively. Mean eGFR values did not differ across categories; mean values and 95\% CI were 56 (53.5-58.4), 58 (56.5-59.5) and $58(57.2-58.7) \mathrm{mL} / \mathrm{min} / 1.73 \mathrm{~m}^{2}$, respectively. Figure $2 \mathrm{~b}$ shows mean values of eGFR in participants with and without albuminuria in groups with a low and high score on the GDS. In participants with albuminuria, mean values of eGFR did not differ between participants with a low $(n=517)$ and high score $(n=84)$; mean values and $95 \%$ CI were 45.5 (44.1-47) and 46.4 (42.7-50.1) $\mathrm{mL} / \mathrm{min} / 1.73 \mathrm{~m}^{2}$. In participants without albuminuria, mean values of eGFR did also not differ between groups. Mean eGFR was $58 \mathrm{~mL} / \mathrm{min} / 1.73 \mathrm{~m}^{2}$ (95\%CI $57.3-58.7$ ) in participants with a low score $(n=1419)$ and $57.1 \mathrm{~mL} /$ $\mathrm{min} / 1.73 \mathrm{~m}^{2}$ (95\% CI 55.4-58.8) in participants with a high score on the GDS $(n=232)$.

\section{Discussion}

Within the framework of the SCOPE study, a large observational cohort including persons aged 75 years and older, we found that early or advanced kidney disease seemed to have no negative influence on cognition or mood in late life. Also, no differences in kidney function were observed in participants with and without cognitive impairment and in those with and without depressive symptoms. The presence of albuminuria did not influence our results.

We found that participants in progressively later stages of CKD were more likely to have risk factors for (cerebral) small vessel disease, such as hypertension and diabetes mellitus, than participants in earlier stages [6]. 
Table 2 Baseline characteristics across stages of chronic kidney disease (CKD)

\begin{tabular}{|c|c|c|c|c|c|}
\hline & \multicolumn{4}{|l|}{ Stages of CKD } & \multirow{2}{*}{$\begin{array}{l}p- \\
\text { value }\end{array}$} \\
\hline & Stage $1-2(n=830)$ & Stage $3 \mathrm{~A}(n=862)$ & Stage $3 B(n=419)$ & Stage $4(n=141)$ & \\
\hline Age, years & $78.6(76.5-81.1)$ & $79.6(77.3-83.1)$ & $81(78.2-85.1)$ & $81(77.7-84.8)$ & $<0.001$ \\
\hline Women, \% & 61.1 & 56.8 & 47.7 & 40.4 & $<0.001$ \\
\hline Living alone, \% & 23.1 & 23.7 & 26 & 32.6 & ns \\
\hline Education, years & $12(8-16)$ & $11.5(8-15)$ & $10(8-13)$ & $8(8-12)$ & $<0.001$ \\
\hline \multicolumn{6}{|l|}{ Smoking status, } \\
\hline Current smoker, \% & 5.5 & 3.6 & 4.1 & 3.5 & ns \\
\hline Former smoker, $\%$ & 35.7 & 40 & 44.3 & 44.9 & 0.017 \\
\hline Alcohol $\geq 1$ unit a day, $\%$ & 26.6 & 24.2 & 27.2 & 24.8 & ns \\
\hline BMI, $\mathrm{kg} / \mathrm{m}^{2}$ & $26.7(24.2-29.6)$ & $27.4(24.8-30.6)$ & $27.7(25.3-31.0)$ & $27.7(24.9-31.4)$ & $<0.001$ \\
\hline ADL-independent, $\%$ & 97.1 & 95.7 & 92.1 & 90.1 & $<0.001$ \\
\hline iADL-independent, \% & 66 & 79.5 & 72.3 & 70.2 & $<0.001$ \\
\hline MMSE, score & $29(27-30)$ & $29(27-30)$ & $29(27-30)$ & $30(28-30)$ & 0.001 \\
\hline MMSE < 24, \% & 6.4 & 7.7 & 9.3 & 5 & $n s^{*}$ \\
\hline GDS, score & $2(1-4)$ & $2(1-4)$ & $2(1-4)$ & $2(1-4)$ & ns \\
\hline $\mathrm{GDS}>5, \%$ & 13 & 15 & 14.1 & 14.2 & ns \\
\hline Hypertension, \% & 66.3 & 77.6 & 89.3 & 96.5 & $<0.001$ \\
\hline Diabetes mellitus, $\%$ & 19.3 & 22.6 & 38.2 & 36.2 & $<0.001$ \\
\hline $\mathrm{TIA}, \%$ & 8 & 8.4 & 11.2 & 7.8 & ns \\
\hline Stroke, \% & 4.3 & 5.7 & 7.9 & 9.2 & 0.023 \\
\hline Atrial fibrillation, \% & 9.3 & 16.6 & 22 & 22.7 & $<0.001$ \\
\hline COPD, \% & 8.9 & 11.3 & 16.7 & 17.7 & $<0.001$ \\
\hline Cancer, \% & 14.2 & 17.2 & 20.8 & 24.8 & 0.002 \\
\hline $\mathrm{CHF}, \%$ & 8.4 & 17.9 & 23.9 & 34.8 & $<0.001$ \\
\hline Vascular disease, $\%$ & 10.8 & 12.3 & 16.5 & 13.5 & 0.042 \\
\hline CIRS-G, total score & $7(4-10)$ & $7(5-10)$ & $10(7-14)$ & $11.5(8.3-15)$ & $<0.001$ \\
\hline CIRS-G, severity index & $1.4(1.2-1.7)$ & $1.5(1.2-1.8)$ & $1.7(1.3-1.9)$ & $1.8(1.5-2)$ & $<0.001$ \\
\hline eGFR-BIS, mL/min/1.73 $\mathrm{m}^{2}$ & $67.2(63.6-73.1)$ & $53.8(50.2-56.9)$ & $39.0(34.9-42.3)$ & $24(20.5-27.1)$ & $<0.001$ \\
\hline $\mathrm{ACR}, \mathrm{mg} / \mathrm{g}$ & $8.3(1.9-18.7)$ & $9.4(3.3-24.3)$ & $24.5(7-121.8)$ & 161 (53.7-1006) & $<0.001$ \\
\hline Albuminuria, \% & 14 & 20.8 & 45.6 & 81.6 & $<0.001$ \\
\hline
\end{tabular}

Continuous variables are expressed as median (IQR)

Abbreviations: BMI Body Mass Index, (i) ADL (instrumental) Activities of Daily Living, MMSE Mini-Mental State Examination, GDS Geriatric Depression Scale, TIA Transient Ischemic Attack, COPD Chronic Obstructive Pulmonary Disease, CHF Congestive Heart Failure, CIRS-G Cumulative Illness Rating Scale for Geriatrics, eGFRBIS Estimated Glomerular Filtration Rate, ACR Albumin-to-Creatinine Ratio *Adjusted for age, sex and education

Also other conditions closely linked to (cerebral) small vessel disease were more prevalent in those in advanced stages of CKD than in those in early stages, such as atrial fibrillation, congestive heart failure and history of stroke [31-33]. The presence of these risk factors is suggested to mediate associations between the kidney and the brain [6], however, we were not able to confirm our prior hypothesis. Previous cross-sectional studies investigated the association between CKD and cognitive impairment in older persons. Most studies investigated this topic in specific populations, such as women with coronary artery disease [34] or only men [35]. In a large sample in the United States of America, it was found that lower levels of kidney function were associated with an increased prevalence of cognitive impairment, assessed with a six-item cognitive screening [36]. In this study, kidney function was assessed by the Modification of Diet in Renal Disease Study (MDRD) equation, an equation that is less reliable than the BIS1 equation in older persons [19], therefore, it is possible that the method used to assess eGFR might have induced bias.

To the best of our knowledge, this is the first crosssectional study investigating the possible association between kidney function and depressive symptoms in a 


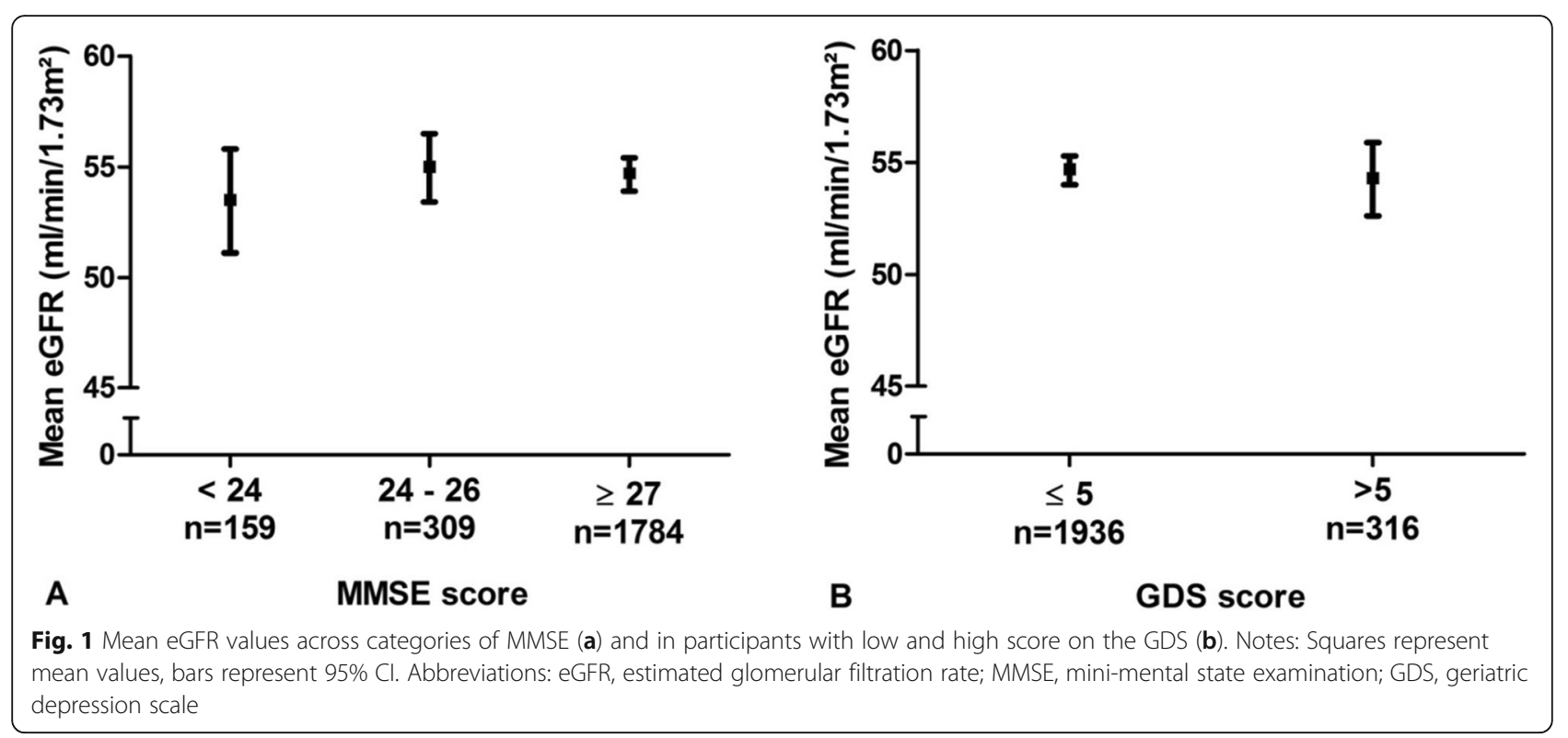

large study population of community dwelling persons in late life within a broad range of eGFR. In a recent meta-analysis, lower kidney function was associated with higher prevalence of depression [17]. However, only two studies investigated persons with a mean age above 70 years [37, 38]. In a Korean population-based cohort study including almost 1000 participants aged 65 years and older, it was found that an eGFR $<45 \mathrm{~mL} / \mathrm{min}$ was associated with poor physical quality of life but not with mental health [37]. In older patients admitted with congestive heart failure [38], depression was more prevalent among those with than those without severe CKD $(<30 \mathrm{vs} \geq 30 \mathrm{~mL} / \mathrm{min})$. However, since this is a very specific study population, these results cannot be completely compared to our results. Both previous studies used different tools to investigate depressive symptoms $[37,38]$ : the Short Form 36 (SF-36) health survey and the Beck Depression Inventory (BDI), respectively. Therefore it cannot be excluded that the different methods used might have affected the reported results.

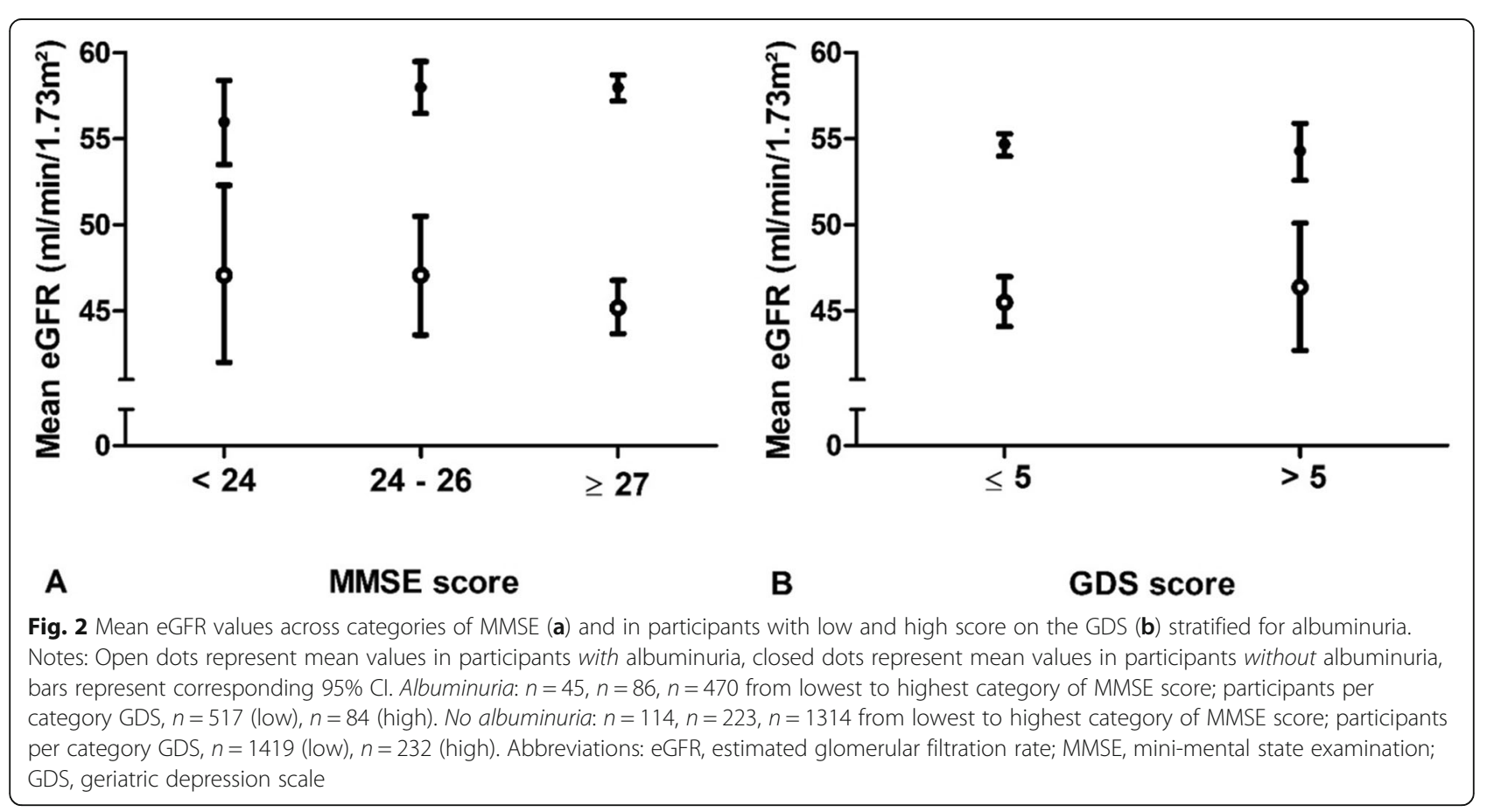


A possible explanation for our findings could lie in the selection of our study population. Eligible persons were community-dwelling, 75 years and older and referred to the outpatient clinics of participating institutions, which might have resulted in a ceiling effect. Almost all participants were under regular medical monitoring at outpatient services, which might have resulted in a better control of cardiovascular risk factors. Therefore, the kidney function and its mediators might no longer be related on the outcomes of interest. One might argue whether kidney function is relevant for the brain (cognition and mood) in the outpatient with multimorbidity in late life or whether all comorbidities in the older outpatient equally contribute to (disturbances in) cognition and mood.

Some limitations of the study need to be discussed. First, the cross sectional design does not allow to investigate causal inferences. Second, persons with relatively good cognition and mood are more likely to volunteer to participate in this study with an extensive protocol, which might have affected our results. Third, persons with end stage renal disease were not included in this study. It might be speculated that mood and cognition are not yet affected in stages 1-4 of CKD due to the asymptomatic nature of the disease. Fourth, there might be a survival bias in which individuals with CKD in advanced stages and possibly cognitive impairment and/or depressive symptoms already died and therefore were not included. Fifth, our study did not include a direct measurement of GFR, however, we used the eGFR-BIS1 equation which is one of the most reliable creatininebased equations at older age [19]. Eventually, we used the MMSE to assess cognition and the GDS-15 to assess depressive symptoms. We cannot exclude that performing a complete neuropsychological evaluation or using other screening tools might have given different results and might be able to confirm our hypotheses. Ideally, such a complete neuropsychological evaluation can be used in future studies.

This study also has strengths. We have studied a large real-world population of older outpatients in 7 different countries across Europe, therefore our findings can be extrapolated to a large population of European citizens. Second, information on kidney function, cognition and mood were obtained systematically in all participating centers, which makes the results highly reliable.

\section{Conclusions}

In community-dwelling older persons in more advanced stages of CKD, cognitive impairment and depressive symptoms were not more prevalent than in older persons without or in earlier stages of CKD. Kidney function was comparable in those with and without any signs of cognitive or mood disorders. The identification of
CKD as modifiable risk factor for cognitive impairment and depressive symptoms in late life might be relevant in order to optimize therapeutic strategies. Longitudinal studies might give additional information on the possible effect of kidney function on mental health in late life. An ongoing prospective SCOPE study is now conducted to investigate effects of CKD progression on these variables.

\section{Abbreviations}

ACR: Albumin-to-Creatinin Ratio; ADL: Activities of Daily Living; BDI: Beck Depression Inventory; BIS1: Berlin Initiative Study 1; CGA: Comprehensive Geriatric Assessment; CIRS-G: Cumulative Illness Rating Scale for Geriatrics; CKD: Chronic Kidney Disease; eGFR: Estimated Glomerular Filtration Rate: GDS(- 15): Geriatric Depression Scale (15 items); GFR: Glomerular Filtration Rate; IADL: Instrumental Activities of Daily living; KDIGO: Kidney Disease Improving Global Outcomes; MDRD: Modification of Diet in Renal Disease Study; MMSE: Mini-Mental State Examination; SCOPE: Screening for CKD among Older People across Europe; SF-36: Short Form 36

\section{Acknowledgements}

SCOPE study investigators

Coordinating center, Fabrizia Lattanzio, Italian National Research Center on Aging (INRCA), Ancona, Italy - Principal Investigator. Andrea Corsonello, Silvia Bustacchini, Silvia Bolognini, Paola D'Ascoli, Raffaella Moresi, Giuseppina Di Stefano, Cinzia Giammarchi, Anna Rita Bonfigli, Roberta Galeazzi, Federica Lenci, Stefano Della Bella, Enrico Bordoni, Mauro Provinciali, Robertina

Giacconi, Cinzia Giuli, Demetrio Postacchini, Sabrina Garasto, Annalisa Cozza, Francesco Guarasci, Sonia D'Alia - Italian National Research Center on Aging (INRCA), Ancona, Fermo and Cosenza, Italy - Coordinating staff. Romano Firmani, Moreno Nacciariti, Mirko Di Rosa, Paolo Fabbietti - Technical and statistical support.

Participating centers

- Department of Internal Medicine, Medical University of Graz, Austria: Gerhard Hubert Wirnsberger, Regina Elisabeth Roller-Wirnsberger, Carolin Herzog, Sonja Lindner

- Section of Geriatric Medicine, Department of Internal Medicine, Erasmus MC, University Medical Center Rotterdam, The Netherlands: Francesco Mattace-Raso, Lisanne Tap, Gijsbertus Ziere, Jeannette Goudzwaard. - Department of Geriatrics, Healthy Ageing Research Centre, Medical University of Lodz, Poland: Tomasz Kostka, Agnieszka Guligowska, Łukasz Kroc, Bartłomiej K Sołtysik, Małgorzata Pigłowska, Agnieszka Wójcik, Zuzanna Chrząstek, Natalia Sosowska, Anna Telążka, Joanna Kostka, Elizaveta Fife, Katarzyna Smyj, Kinga Zel.

- The Recanati School for Community Health Professions at the faculty of Health Sciences at Ben-Gurion University of the Negev, Israel: Rada ArtziMedvedik, Yehudit Melzer, Mark Clarfield, Itshak Melzer; and Maccabi Healthcare services southern region, Israel: Rada Artzi-Medvedik, Ilan Yehoshua, Yehudit Melzer.

- Geriatric Unit, Internal Medicine Department and Nephrology Department, Hospital Universitari de Bellvitge, Institut d'Investigació Biomèdica de

Bellvitge - IDIBELL, L'Hospitalet de Llobregat, Barcelona, Spain: Francesc Formiga, Rafael Moreno-González, Xavier Corbella, Yurema Martínez, Carolina Polo, Josep Maria Cruzado.

- Department of Geriatric Medicine, Hospital Clínico San Carlos, Madrid: Pedro Gil Gregorio, Sara Laínez Martínez, Mónica González Alonso, Jose A. Herrero Calvo, Fernando Tornero Molina, Lara Guardado Fuentes, Pamela Carrillo García, María Mombiedro Pérez.

- Department of General Internal Medicine and Geriatrics, Krankenhaus Barmherzige Brüder Regensburg and Institute for Biomedicine of Aging Friedrich-Alexander-Universität Erlangen-Nürnberg, Germany: Alexandra Renz, Susanne Muck, Stephan Theobaldy, Andreas Bekmann, Revekka Kaltsa, Sabine Britting, Robert Kob, Christian Weingart, Ellen Freiberger, Cornel Sieber. - Department of Medical Sciences, Uppsala University, Sweden: Johan Ärnlöv, Axel Carlsson, Tobias Feldreich.

Scientific advisory board (SAB)

Roberto Bernabei, Catholic University of Sacred Heart, Rome, Italy

Christophe Bula, University of Lausanne, Switzerland

Hermann Haller, Hannover Medical School, Hannover, Germany 
Carmine Zoccali, CNR-IBIM Clinical Epidemiology and Pathophysiology of Renal Diseases and Hypertension, Reggio Calabria, Italy

Data and Ethics Management Board (DEMB)

Dr. Kitty Jager, University of Amsterdam, The Netherlands

Dr. Wim Van Biesen, University Hospital of Ghent, Belgium

Paul E. Stevens, East Kent Hospitals University NHS Foundation Trust,

Canterbury, United Kingdom. We thank the BioGer IRCCS INRCA Biobank for the collection of the SCOPE samples.

\section{About this supplement}

This article has been published as part of BMC Geriatrics Volume 20 Supplement 1 2020: The Screening for Chronic Kidney Disease among Older People across Europe (SCOPE) project: findings from cross-sectional analysis. The full contents of the supplement are available at https://bmcgeriatr.biomedcentral.com/articles/supplements/volume-20-supplement-1.

\section{Authors' contributions}

LT, FMR: participated in study protocol design, data collection, leading statistical analysis, manuscript drafting and revision. AC: conceived the study, coordinated study protocol and data collection, participated in statistical analysis and manuscript revision. PF: participated in statistical analysis and manuscript revision. FF, RMG, JA, ACa, RRW, GW, GZ, EF, CS, TK, AG, PG, SLM, RAM, IY: participated in study protocol design, data collection, and manuscript revision. FL: conceived the study, coordinated study protocol and data collection, participated in manuscript revision. All authors read and approved the final manuscript.

\section{Funding}

SCOPE study and publication costs are funded by the European Union Horizon 2020 program, under the Grant Agreement n 634869. Funding body had no role in the design of the study and collection, analysis, and interpretation of data and in writing the manuscript.

\section{Availability of data and materials}

The datasets generated and/or analysed during the current study are available in the SCOPE repository (www.scopeproject.eu).

\section{Ethics approval and consent to participate}

Ethics approvals have been obtained by Ethics Committees in participating institutions as follows:

- Italian National Research Center on Aging (INRCA), Italy, \#2015 0522 IN, January 27, 2016.

- University of Lodz, Poland, \#RNN/314/15/KE, November 17, 2015.

- Medizinische Universität Graz, Austria, \#28-314 ex 15/16, August 5, 2016

- Erasmus MC University Medical Center Rotterdam, The Netherland, \#MEC-

2016-036 - \#NL56039.078.15, v.4, March 7, 2016.

- Hospital Clínico San Carlos, Madrid, Spain, \# 15/532-E_BC, September 16 2016

- Bellvitge University Hospital Barcellona, Spain, \#PR204/15, January 29, 2016.

- Friedrich-Alexander University Erlangen-Nürnberg, Germany, \#340_15B, January 21, 2016.

- Helsinki committee in Maccabi Healthcare services, Bait Ba-lev, Bat Yam, Israel, \#45/2016, July 24, 2016.

All patients signed a written informed consent to be enrolled.

\section{Consent for publication}

Not applicable.

\section{Competing interests}

The authors declare that they have no competing interests.

\section{Author details}

'Department of Internal Medicine, Section of Geriatric Medicine, Erasmus MC, University Medical Center Rotterdam, Rotterdam, The Netherlands. ${ }^{2}$ Italian National Research Center on Aging (IRCCS INRCA), Ancona, Fermo and Cosenza, Italy. ${ }^{3}$ Geriatric Unit, Internal Medicine Department, Bellvitge University Hospital-IDIBELL-L'Hospitalet de Llobregat, Barcelona, Spain. ${ }^{4}$ Department of Medical Sciences, Uppsala University, Uppsala, Sweden. ${ }^{5}$ School of Health and Social Studies, Dalarna University, Falun, Sweden. ${ }^{6}$ Division of Family Medicine, Department of Neurobiology, Care Sciences and Society, Karolinska Institutet, Stockholm, Sweden. ${ }^{7}$ Department of
Internal Medicine, Medical University of Graz, Graz, Austria. ${ }^{8}$ Department of Internal Medicine-Geriatrics, Institute for Biomedicine of Aging (IBA), Friedrich-Alexander Universität Erlangen-Nürnberg, Erlangen, Germany. ${ }^{9}$ Department of Geriatrics, Healthy Ageing Research Centre, Medical University of Lodz, Lodz, Poland. ${ }^{10}$ Geriatric Department, Hospital Clinico San Carlos, Madrid, Spain. ${ }^{11}$ The Recanati School for Community Health Professions at the faculty of Health Sciences, Ben-Gurion University of the Negev, Beer-sheva, Israel. ${ }^{12}$ Maccabi Health Organization, Negev district, Tel Aviv-Yafo, Israel. ${ }^{13}$ Laboratory of Geriatric Pharmacoepidemiology and Biostatistics, IRCCS INRCA, Via S. Margherita 5, 60124 Ancona, Italy.

\section{Received: 10 August 2020 Accepted: 13 August 2020}

Published: 2 October 2020

\section{References}

1. Stevens PE, O'Donoghue DJ, de Lusignan S, Van Vlymen J, Klebe B, Middleton R, Hague N, New J, Farmer CK. Chronic kidney disease management in the United Kingdom: NEOERICA project results. Kidney Int. 2007;72(1):92-9.

2. Ferri CP, Prince M, Brayne C, Brodaty H, Fratiglioni L, Ganguli M, Hall K, Hasegawa K, Hendrie H, Huang Y, et al. Global prevalence of dementia: a Delphi consensus study. Lancet. 2005;366(9503):2112-7.

3. Isomaa B, Almgren P, Tuomi T, Forsen B, Lahti K, Nissen M, Taskinen MR, Groop L. Cardiovascular morbidity and mortality associated with the metabolic syndrome. Diabetes Care. 2001;24(4):683-9.

4. Gansevoort RT, Correa-Rotter R, Hemmelgarn BR, Jafar TH, Heerspink HJ, Mann JF, Matsushita K, Wen CP. Chronic kidney disease and cardiovascular risk: epidemiology, mechanisms, and prevention. Lancet. 2013;382(9889): 339-52.

5. Knopman D, Boland LL, Mosley T, Howard G, Liao D, Szklo M, McGovern P, Folsom AR. Atherosclerosis risk in communities study I: cardiovascular risk factors and cognitive decline in middle-aged adults. Neurology. 2001;56(1): $42-8$

6. Lau WL, Huisa BN, Fisher M. The cerebrovascular-chronic kidney disease connection: perspectives and mechanisms. Transl Stroke Res. 2017;8(1):6776.

7. Coresh J, Selvin E, Stevens LA, Manzi J, Kusek JW, Eggers P, Van Lente F, Levey AS. Prevalence of chronic kidney disease in the United States. JAMA. 2007;298(17):2038-47.

8. Vermeer SE, Hollander M, van Dijk EJ, Hofman A, Koudstaal PJ, Breteler MM, Rotterdam Scan S. Silent brain infarcts and white matter lesions increase stroke risk in the general population: the Rotterdam Scan Study. Stroke. 2003;34(5):1126-9.

9. van Dijk EJ, Prins ND, Vrooman HA, Hofman A, Koudstaal PJ, Breteler MMB. Progression of cerebral small vessel disease in relation to risk factors and cognitive consequences: Rotterdam Scan study. Stroke. 2008;39(10):2712-9.

10. van Sloten TT, Protogerou AD, Henry RMA, Schram MT, Launer $L$, Stehouwer CDA. Association between arterial stiffness, cerebral small vessel disease and cognitive impairment: a systematic review and meta-analysis. Neurosci Biobehav Rev. 2015:53:121-30.

11. Manschot SM, Biessels GJ, de Valk H, Algra A, Rutten GE, van der Grond J, Kappelle LJ. Utrecht Diabetic Encephalopathy Study G: metabolic and vascular determinants of impaired cognitive performance and abnormalities on brain magnetic resonance imaging in patients with type 2 diabetes. Diabetologia. 2007;50(11):2388-97.

12. van Sloten $\Pi T$, Sigurdsson $S$, van Buchem $M A$, Phillips $C L$, Jonsson PV, Ding J, Schram MT, Harris TB, Gudnason V, Launer L. Cerebral small vessel disease and association with higher incidence of depressive symptoms in a general elderly population: the AGES-Reykjavik study. Am J Psychiatr. 2015; 172(6):570-8.

13. Burn DJ, Bates D. Neurology and the kidney. J Neurol Neurosurg Psychiatry. 1998;65(6):810-21

14. Sastre M, Calero M, Pawlik M, Mathews PM, Kumar A, Danilov V, Schmidt SD, Nixon RA, Frangione B, Levy E. Binding of cystatin C to Alzheimer's amyloid beta inhibits in vitro amyloid fibril formation. Neurobiol Aging. 2004;25(8): 1033-43.

15. Yaffe K, Lindquist K, Shlipak MG, Simonsick E, Fried L, Rosano C, Satterfield S, Atkinson H, Windham BG, Kurella-Tamura M. Cystatin $C$ as a marker of cognitive function in elders: findings from the health $A B C$ study. Ann Neurol. 2008;63(6):798-802. 
16. Etgen T, Chonchol M, Forstl H, Sander D. Chronic kidney disease and cognitive impairment: a systematic review and meta-analysis. Am J Nephrol. 2012;35(5):474-82.

17. Palmer S, Vecchio M, Craig JC, Tonelli M, Johnson DW, Nicolucci A, Pellegrini F, Saglimbene V, Logroscino G, Fishbane S, et al. Prevalence of depression in chronic kidney disease: systematic review and meta-analysis of observational studies. Kidney Int. 2013;84(1):179-91.

18. Corsonello A, Tap L, Roller-Wirnsberger R, Wirnsberger G, Zoccali C, Kostka T, Guligowska A, Mattace-Raso F, Gil P, Fuentes LG, et al. Design and methodology of the screening for CKD among older patients across Europe (SCOPE) study: a multicenter cohort observational study. BMC Nephrol. 2018;19(1):260.

19. Schaeffner ES, Ebert N, Delanaye P, Frei U, Gaedeke J, Jakob O, KuhImann MK, Schuchardt M, Tolle M, Ziebig R, et al. Two novel equations to estimate kidney function in persons aged 70 years or older. Ann Intern Med. 2012; 157(7):471-81.

20. Kidney Disease: Improving Global Outcomes (KDIGO) CKD Work Group: KDIGO 2012 Clinical Practice Guideline for the Evaluation and Management of Chronic Kidney Disease. Kidney Int Suppl. 2013(3):1-150.

21. Folstein MF, Folstein SE, McHugh PR. "Mini-mental state". A practical method for grading the cognitive state of patients for the clinician. J Psychiatr Res. 1975;12(3):189-98.

22. Tsoi KK, Chan JY, Hirai HW, Wong SY, Kwok TC. Cognitive tests to detect dementia: a systematic review and meta-analysis. JAMA Intern Med. 2015; 175(9):1450-8.

23. Mitchell AJ. A meta-analysis of the accuracy of the mini-mental state examination in the detection of dementia and mild cognitive impairment. J Psychiatr Res. 2009:43(4):411-31.

24. O'Bryant SE, Humphreys JD, Smith GE, Ivnik RJ, Graff-Radford NR, Petersen $\mathrm{RC}$, Lucas JA. Detecting dementia with the mini-mental state examination in highly educated individuals. Arch Neurol. 2008;65(7):963-7.

25. Sheikh Jl, Yesavage JA. Geriatric depression scale (GDS): recent evidence and development of a shorter version. Clin Gerontol. 1986;5(1-2):165-73.

26. Wancata J, Alexandrowicz R, Marquart B, Weiss M, Friedrich F. The criterion validity of the geriatric depression scale: a systematic review. Acta Psychiatr Scand. 2006;114(6):398-410.

27. Krishnamoorthy $Y$, Rajaa S, Rehman T. Diagnostic accuracy of various forms of geriatric depression scale for screening of depression among older adults: systematic review and meta-analysis. Arch Gerontol Geriatr. 2020;87: 104002.

28. Conwell Y, Forbes NT, Cox C, Caine ED. Validation of a measure of physical illness burden at autopsy: the cumulative illness rating scale. J Am Geriatr Soc. 1993:41(1):38-41.

29. Katz S, Ford AB, Moskowitz RW, Jackson BA, Jaffe MW. Studies of illness in the aged. The index of Adl: a standardized measure of biological and psychosocial function. JAMA. 1963;185:914-9.

30. Lawton MP, Brody EM. Assessment of older people: self-maintaining and instrumental activities of daily living. Gerontologist. 1969;9(3):179-86.

31. Mayasi Y, Helenius J, McManus DD, Goddeau RP Jr, Jun-O'Connell AH, Moonis M, Henninger N. Atrial fibrillation is associated with anterior predominant white matter lesions in patients presenting with embolic stroke. J Neurol Neurosurg Psychiatry. 2018;89(1):6-13.

32. Breteler MM, van Swieten JC, Bots ML, Grobbee DE, Claus JJ, van den Hout $J$ H, van Harskamp F, Tanghe HL, de Jong PT, van Gijn J, et al. Cerebral white matter lesions, vascular risk factors, and cognitive function in a populationbased study: the Rotterdam Study. Neurology. 1994;44(7):1246-52.

33. Vogels RL, van der Flier WM, van Harten B, Gouw AA, Scheltens P, SchroederTanka JM, Weinstein HC. Brain magnetic resonance imaging abnormalities in patients with heart failure. Eur J Heart Fail. 2007;9(10):1003-9.

34. Kurella M, Yaffe K, Shlipak MG, Wenger NK, Chertow GM. Chronic kidney disease and cognitive impairment in menopausal women. Am J Kidney Dis. 2005;45(1):66-76.

35. Yang AC, Tsai SJ, Yeh HL, Chen JY, Liou YJ, Hwang JP, Hong CJ. Association between renal function and cognitive performance in elderly communitydwelling men without dementia. J Am Geriatr Soc. 2010;58(10):2046-8.

36. Kurella Tamura M, Wadley V, Yaffe K, McClure LA, Howard G, Go R, Allman RM, Warnock DG, McClellan W. Kidney function and cognitive impairment in US adults: the reasons for geographic and racial differences in stroke (REGARDS) study. Am J Kidney Dis. 2008;52(2):227-34.

37. Chin HJ, Song YR, Lee JJ, Lee SB, Kim KW, Na KY, Kim S, Chae DW. Moderately decreased renal function negatively affects the health-related quality of life among the elderly Korean population: a population-based study. Nephrol Dial Transplant. 2008;23(9):2810-7.

38. Hedayati SS, Jiang W, O'Connor CM, Kuchibhatla M, Krishnan KR, Cuffe MS, Blazing MA, Szczech LA. The association between depression and chronic kidney disease and mortality among patients hospitalized with congestive heart failure. Am J Kidney Dis. 2004;44(2):207-15.

\section{Publisher's Note}

Springer Nature remains neutral with regard to jurisdictional claims in published maps and institutional affiliations.
Ready to submit your research? Choose BMC and benefit from:

- fast, convenient online submission

- thorough peer review by experienced researchers in your field

- rapid publication on acceptance

- support for research data, including large and complex data types

- gold Open Access which fosters wider collaboration and increased citations

- maximum visibility for your research: over $100 \mathrm{M}$ website views per year

At BMC, research is always in progress.

Learn more biomedcentral.com/submissions 